Check for updates

Cite this: RSC Adv., 2017, 7, 49114

Received 8th August 2017

Accepted 22nd September 2017

DOI: 10.1039/c7ra08769a

rsc.li/rsc-advances

\section{Side chain length impacting thermal transitions and water uptake of acrylate-maleimide copolymers with pendent catechols $\uparrow$}

\author{
M. A. Bartucci, E. Napadensky, (D) J. L. Lenhart and J. A. Orlicki (D)* \\ To elucidate how $T_{\mathrm{g}}$ affects adhesion of synthetic polymers containing pendent catechols, a series of \\ poly(acrylate-r-(1-[2-(3,4-dihydroxyphenyl)ethyl]-maleimides)) (PAMs) have been synthesized that vary in \\ their acrylate side chain length. During characterization, a thermal transition was observed that over- \\ rode the anticipated trends in $T_{\mathrm{g}}$. Careful material handling and analysis using TGA-MS techniques led to \\ the realization that water absorption is an intrinsic characteristic of this class of polymer and must be \\ considered as applications are developed.
}

The mussel foot proteins (mfps) of mytilidae are often discussed as natural adhesives due to their ability to adhere to a variety of surfaces in aquatic or wet climates. ${ }^{1-3}$ Many commercial adhesives exhibit accelerated failure rates in high moisture environments via delamination of the polymer from the substrate..$^{4,5}$ Due to the mfps effectiveness in dirty and wet conditions, ${ }^{6-12}$ substantial investigation into the chemical composition and residue sequence has been pursued. From a residue composition vantage point, approximately $20-30 \%$ of the interfacial mfp-3 and -5 comprised the 3,4-dihydroxyphenylalanine (Dopa) residue. ${ }^{13,14}$ The Dopa residue allows for multifunctional chemistry at the interface due to a pendent catechol (1,2-dihydroxybenzene) that can chemically bind to a surface acting as a natural surface primer, covalently crosslink to aid in repelling water at the interface, or chelate/complex to metals to improve toughness of the protein, all of which result in improved adhesion. ${ }^{15-20}$

Inspiration from the multifunctional chemistry of the catechol moiety has recently led to its incorporation into synthetic $^{21-25}$ and biomimetic ${ }^{26-28}$ polymers. Some of these initial studies have investigated water-resistant adhesives, ${ }^{29,30}$ the use of various oxidants to control covalent crosslinks, ${ }^{31}$ and a wide variety of fillers to improve adhesion/toughness of catechol-containing polymers. ${ }^{32}$ However, fundamental polymer physics questions arise as catechols are incorporated into bulk synthetic adhesives (e.g. polymer mechanics, thermal/UV aging etc.), namely due to the multifunctional chemistry the catechol offers (e.g. hydrogen boding, metal chelating, etc.). One of our goals is to understand how the adhesion of a catechol system is altered by varying the $T_{\mathrm{g}}$ or polarity of the polymer. It would be ideal to synthesize an adhesive that can perform over

U.S. Army Research Laboratory, USA.E-mail: joshua.a.orlicki.civ@mail.mil

$\dagger$ Electronic supplementary information (ESI) available. See DOI: 10.1039/c7ra08769a wide temperature and humidity ranges with minimal effects on the adhesion or mechanics for applications in widely varying climatic conditions.

In an attempt to deconvolute the factors contributing to catechol adhesion, the characteristics such as backbone polarity and glass transition temperature were probed with a series of poly(acrylate-maleimide) copolymers (PAM-1-5) (Table 1). It is well established that controlling acrylate sidechain composition governs the resulting $T_{\mathrm{g}}{ }^{33-35}$ and potential application ${ }^{36}$ of the polymer. We elected to use these relationships to effect control over polarity and $T_{\mathrm{g}}$ of the PAMs by varying the acrylate side chain from a single methyl to a 2-ethylhexyl which acts as an eight carbon surrogate.

Using a free radical polymerization route with a $1: 1$ acrylate to maleimide monomer (1) feed ratio allowed for approximate sequence and composition control of the PAMs. Due to the free radical copolymerization reactivity ratios of similar acrylates and maleimide monomers, ${ }^{37}$ it was expected that the sequence should be alternating with the acrylates $(\mathrm{x})$ having a mildly higher affinity to polymerize with themselves (e.g. xyxy and xxyxxy). Although there are reports that maleimide (y) can homopolymerize under select conditions, ${ }^{38,39}$ the propensity to alternate reaction with the acrylates was preferred. Thus, adjacent catechols in the resulting backbone sequence should be minimized. This is reflected in the final polymer repeat unit stoichiometry $x: y$ ratios $(53: 47)$ observed via ${ }^{1} \mathrm{H}$ NMR as the percentage of maleimide is consistent (within 3\%) for this class of PAMs (ESI-Fig. S1 $\dagger$ ). The catechol spacing, along with the two carbon linker from the succinimide nitrogen, is similar in connectivity to the naturally occurring Dopa residue, and should provide sufficient free volume for potential catechol interaction when adsorbed onto a surface. Also, the mechanics of the polymers should be similar as the $M_{\mathrm{n}}$ for the silyl-protected PAM-1-5 
Table 1 Respective acrylates polymerized with 1 and deprotected to afford PAM 1-5<smiles>[R]OC(=O)C=C</smiles>

1<smiles>[Y]CC(C([R])[R])C1C(=O)N(CCc2ccc(O)c(O)c2)C(=O)C1=C(C)C</smiles>

PAM 1-5

\begin{tabular}{lllllrr}
\hline PAM & $\mathrm{R}$ & Yield $^{a}$ & $M_{\mathrm{n}}{ }^{b}(\mathrm{kDa})$ & $\mathrm{PDI}^{b}$ & $T_{\mathrm{g} \text { wet }}\left({ }^{\circ} \mathrm{C}\right)$ & $T_{\mathrm{g}}\left({ }^{\circ} \mathrm{C}\right)$ \\
\hline $\mathbf{1}$ & Methyl & 67 & 114 & 2.55 & - & 129 \\
$\mathbf{2}$ & Ethyl & 88 & 136 & 3.80 & 76 & $60.2 \pm 1.6$ \\
$\mathbf{3}$ & Butyl & 93 & 134 & 3.85 & 71 & $63.1 \pm 1.7$ \\
$\mathbf{4}$ & Hexyl & 73 & 118 & 3.27 & 63 & $70.8 \pm 0.9$ \\
$\mathbf{5}$ & 2-Ethylhexyl & 69 & 124 & 2.24 & 60 & $83.9 \pm 2.1$ \\
& & & & & $93.8 \pm 2.6$
\end{tabular}

${ }^{a}$ Yields are of deprotected polymers over two steps. ${ }^{b} M_{\mathrm{n}}$ and PDIs were taken of silyl-protected polymers as catechol system appeared to stick to GPC column. ${ }^{c}$ Advancing contact angle with water.

are all above $100 \mathrm{kDa}$, which exceeds the molecular weight of entanglement for acrylates. ${ }^{\mathbf{4 0 , 4 1}}$

The unprotected catechol is a free radical scavenger, so the phenols of monomer 1 (ESI-Scheme S1†) were silane protected to allow for radical propagation. After the polymer was isolated, the protecting groups were removed via addition of small amounts of concentrated hydrochloric acid. While catechol surface binding, adhesion, and the formation of metal-catechol complexes have shown dependence on $\mathrm{pH}$, this relationship is very much dependent on the local media and substrate surface energies. ${ }^{\mathbf{1 0 - 1 2 , 4 2 , 4 3}}$ Under the conditions reported here, if PAM-15 were left in an acidic environment they discolored (oxidized) after a week. To remove excess acid after the deprotection, PAM1-5 were precipitated into water from THF three times. The $\mathrm{pH}$ of the precipitate solution was measured to ensure a range between 6-7. After precipitation into water, PAM-1-5 were dried under vacuum ( 0.17 Torr) for a minimum of five hours to afford off white powders in good to moderate yields.

After the PAMs were isolated and dried on high vacuum for several hours, their thermal properties were assessed using differential scanning calorimetry. The $T_{\mathrm{g}}$ of the PAMs were expected to be governed by monomer composition and the resulting acrylate side chain length; polar PAM-1 and aliphatic -5 were anticipated to represent the high and low bounds of observed $T_{\mathrm{g}}$, respectively, while PAMs-2-4 allowed for an assessment of the shift in polarity and $T_{\mathrm{g}}$ as the acrylate side chain length was incremented by two carbons. The polymers instead exhibited a cluster of $T_{\mathrm{g}}$ values, with a range of $c a .20{ }^{\circ} \mathrm{C}$ when comparing PAM-1 to PAM-5. Polymers PAM-2-4 displayed broadened and depressed $T_{\mathrm{g}}$ as shown in Fig. 1 (dashed lines), as the butyl sidechain PAM-3 was either lengthened (PAM-4) or shortened (PAM-2) by two carbons. There were weak $T_{\mathrm{g}}$ shifts (ca. 5-10 ${ }^{\circ} \mathrm{C}$ ) relative to PAM-3 (Fig. 1, dashed lines). It was postulated that water adsorption, enabled by the free catechol functionality of the PAMs could be causing depressed and broadened thermal transitions when analyzed using DSC.

To investigate whether catechol water uptake was the cause of the depressed $T_{\mathrm{g}}$, the same sample pans were punctured and heated in the oven at $120{ }^{\circ} \mathrm{C}$ overnight under vacuum. Once

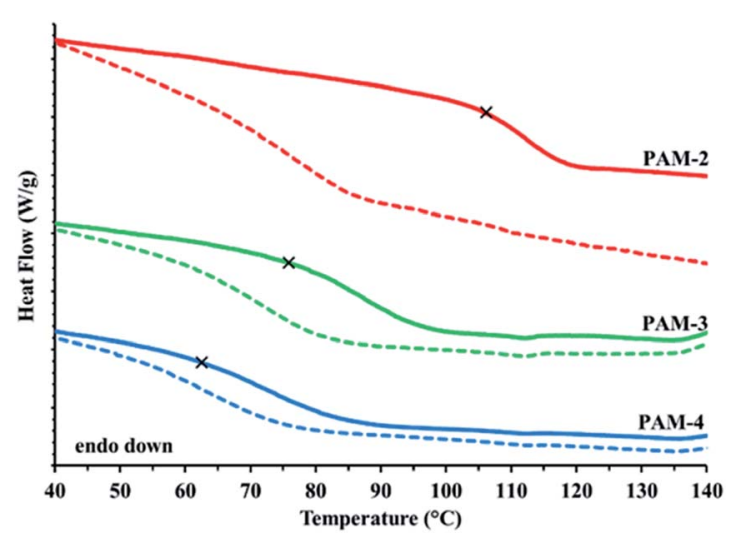

Fig. 1 DSC second heat cycle of 'wet' (dashed lines) and vacuum oven dried (solid lines) of PAM-2 $\left(76{ }^{\circ} \mathrm{C}\right.$ depressed $T_{g}$, red), $-3\left(71{ }^{\circ} \mathrm{C}\right.$ depressed $T_{\mathrm{g}}$, green), and $-4\left(63{ }^{\circ} \mathrm{C}\right.$ depressed $T_{\mathrm{g}}$, blue). Onset temperatures $(x)$ used to calculate dry $T_{\mathrm{g}}$ are 106,76 , and $62{ }^{\circ} \mathrm{C}$ for PAM-2-4 respectively. $T_{\mathrm{g}}$ is a function of backbone polarity for PAM2-4 and wet to dry shifts are correlated to amount of water uptake which is controlled by the polarity of the acrylate side chain. Heat flow is arbitrary as DSC curves have been shifted to make $T_{\mathrm{g}}$ shift easier to view. Heating and cooling rates are $10^{\circ} \mathrm{C} \mathrm{min}^{-1}$. 
dried, the anticipated trend ${ }^{44}$ in $T_{\mathrm{g}}$ with respect to the polarity of the acrylate side chain length was observed (Table 1 and Fig. 1, solid lines). The PAMs reported here have a $T_{\mathrm{g}}$ range of over $60{ }^{\circ} \mathrm{C}$ with PAM-1 at $129^{\circ} \mathrm{C}$ and PAM-5 at $67{ }^{\circ} \mathrm{C}$. Interestingly, the $T_{\mathrm{g}}$ shift from wet to dry for each PAM were proportional to polarity. The ethyl-substituted PAM-2 exhibited an observed shift of about $50{ }^{\circ} \mathrm{C}$ (red curves) while the hexyl-substituted PAM-4 (blue curves) shifted a fifth of that magnitude $\left(\Delta 8^{\circ} \mathrm{C}\right)$. This suggests that the polarity of the acrylate side chain controlled the amount of water that the PAMs adsorbed even though the catechol content was kept constant across these polymers. Further support of the polarity trend was illustrated by the wet and dry observed $T_{\mathrm{g}}$ shifts for the upper and lower bound PAM-1 and -5 (Table 1, ESI-S2 $\uparrow$ ). Of importance to synthetic catechol-containing polymers in general, if the PAMs were left out in ambient conditions for ten days after being dried, these depressed thermal transitions reappeared (ESI$\mathrm{S} 3 \uparrow)$. This suggests that these polymers readily readsorb moisture from the local atmosphere and that these depressed thermal transitions are not solely attributed to the water precipitation in the reaction workup.

To further investigate the origin of the depressed thermal transitions, thermal gravimetric analysis (TGA) coupled with mass spectrometry was undertaken. The low temperature mass loss observed as the PAMs were heated was proportional to the backbone polarity. The largest mass loss attributed to water came from PAMs-1-3 which lost $8-10 \%$ of their mass starting around $100{ }^{\circ} \mathrm{C}$ (Fig. 2a). The onset temperature of water loss for PAMs-1-3 appeared to be a function of polarity $/ T_{\mathrm{g}}$ of the acrylate side chain. The methyl substituted PAM-1 (black curve), the most polar polymer, had the highest onset temperature at about $115{ }^{\circ} \mathrm{C}$. As the chain length was increased to the ethyl PAM-2 (red curve) and butyl substituted PAM-3 (green curve), their onset temperatures decreased to $107^{\circ} \mathrm{C}$ and $83^{\circ} \mathrm{C}$, respectively. The onset temperature of initial mass loss for these more polar PAMs track well with the $T_{\mathrm{g}}$ of the dried polymer backbone, suggesting that water loss was governed by backbone mobility and suggests that the side chain length influenced water uptake. As the acrylate side chain length increased to the hexyl PAM-4 (Fig. 2a, inset, blue curve) and 2-ethylhexyl PAM-5 (Fig. 2a, inset, yellow curve), minimal changes in their mass loss $(<1 \%)$ were observed when heated to $200{ }^{\circ} \mathrm{C}$, which supports that water uptake is proportional to the acrylate side chain length. To assess that the impact of side chain composition on overall polarity, contact angle measurements with water demonstrated the increase in hydrophobicity across the series (Table 1, ESI - Table S1†).

To assess the loss of water in PAM-1 and the relative insensitivity of PAMs-4 and -5, TGA coupled with mass spectrometry was used to characterize the evolved gas during a mass loss event (Fig. 2b). The mass spectrometer was tuned to observe $\mathrm{m} / \mathrm{z}$ 18 (for water), and there was a distinct change in the ion current for PAM-1 at about $130{ }^{\circ} \mathrm{C}$ (black). In contrast, the longer side chain PAM-4 (blue) and -5 (yellow) showed minimal signal over this same temperature range, corresponding to the mass stability exhibited in the thermo-gravimetric scans. Qualitatively, PAM-2 (red) and -3 (green) also showed a loss of water.
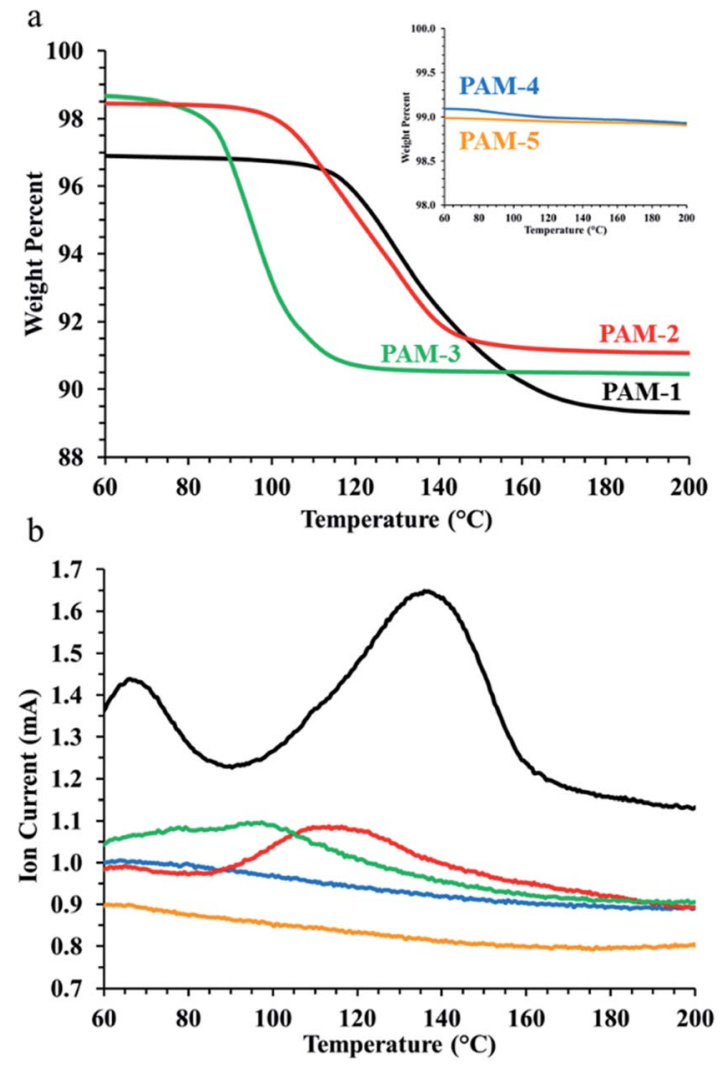

Fig. 2 TGA coupled to mass spectrometry tuned to water at $m / z 18$. (a) TGA of PAM-1 (black), -2 (red), -3 (green), -4 (blue), -5 (yellow). The polar PAM-1-3 release water as the more hydrophobic -4 and -5 show minimal water loss at $100^{\circ} \mathrm{C}$. Note: the scale was changed on the inset to illustrate minimal mass loss of PAM -4 and -5 . Weight percent does not start at $100 \%$ due to an initial ramp rate of $0.5^{\circ} \mathrm{C} \mathrm{min}-1$ to $50{ }^{\circ} \mathrm{C}$ to remove loosely bound water on the PAMs. (b) Mass spectrometry of the exhaust from the TGA experiments tuned to $\mathrm{m} / \mathrm{z} 18$ (water). PAM-1 (black) shows water coming off around $130{ }^{\circ} \mathrm{C}$ while PAM-4 (blue) and -5 (yellow) show no water loss. PAM-2 (red) and -3 (green) show qualitative water loss.

These TGA-MS ion current measurements are not quantitative as water in the atmosphere and purge gas (nitrogen tower bleed gas) can cause variation in peak height and background signal. The ambient water was observed in the first MS peak at $65^{\circ} \mathrm{C}$ for PAM-1 and frustrated the establishment of a true baseline for all the TGA-MS measurements even after initial ramp rate of $0.5^{\circ} \mathrm{C} \min ^{-1}$ to $50{ }^{\circ} \mathrm{C}$. However, the onset temperatures of water loss are consistent and repeatable for the PAMs-1-3 (within $5{ }^{\circ} \mathrm{C}$ ) as well as the mass spectrometry temperature maxima which corresponds to the peak center at half the height of the sigmoidal TGA curve. The onset of water loss also corresponds roughly with the $T_{\mathrm{g}}$ observed for dry samples in the DSC, suggesting that the water loss may be associated with the onset of segmental motion of the polymer chain to enable the dissociation of the water. The intrinsic flexibility of the PAM backbone should be consistent across the library, as they are compositionally similar with essentially alternating acrylate and maleimide comonomers. The variation in thermal properties arises from the aliphatic side-chains of the acrylate monomer, where 
the increasing length of aliphatic side chain results in increasing volume fraction at the same mole fraction of composition. The result is that the methyl side chain of PAM-1 exhibits the stiffness of the acrylate-maleimide backbone while PAM-5 results in significant plasticization or available freevolume, resulting in an observed depression of $T_{\mathrm{g}}{ }^{45,46}$ This library of polymers also suggests that catechol containing polymers may be prepared that exhibit good resistance to water uptake if the correct comonomers are selected. This could have implications to the broader community as the exploration of catechol applications expands.

In sum, a new class of bioinspired catechol based polymers have been synthesized in hopes to elucidate the effects of $T_{\mathrm{g}}$ and backbone polarity on adhesion. As the PAMs polarity was altered, the amount of water present was found to be inversely proportional to the acrylate side chain length. This was reflected by the minimal water uptake of PAM-4 and $\mathbf{- 5}$ which have smaller shifts in the observed $T_{\mathrm{g}}$ before and after drying as opposed to PAM-1-3 which have larger shifts due to higher water content. It is important to note that water uptake of the PAMs could have a significant effect on the fundamental polymer physics properties (e.g. storage modulus, yield strength). Hence, an attempt to understand how water alters these fundamental mechanics is currently ongoing so accurate conclusions can be garnered from future adhesion testing. Morphology characterization of the PAMs, along with mechanical testing, is currently ongoing to elucidate $T_{\mathrm{g}}$ and water effects that control adhesion of these synthetic catechol systems.

\section{Conflicts of interest}

There are no conflicts to declare.

\section{Acknowledgements}

This research was supported in part by an appointment to the Postgraduate Research Participation Program at the U.S. Army Research Laboratory administered by the Oak Ridge Institute for Science and Education through an interagency agreement between the U.S. Department of Energy and USARL.

\section{Notes and references}

1 D. J. Crisp, G. Walker, G. A. Young and A. B. Yule, Adhesion and Substrate Choice in Mussels and Barnacles, J. Colloid Interface Sci., 1985, 104, 40-50.

2 M. Weigemann, Adhesion in Blue Mussels (Mytilus edulis) and Barnacles (genus Balanus): Mechanisms and Technical Applications, Aquat. Sci., 2005, 67, 167-176.

3 H. G. Silverman and F. F. Roberto, Understanding Marine Mussel Adhesion, Mar. Biotechnol., 2007, 9, 661-681.

4 E. M. Petrie, Handbook of Plastics and Elastomers, McGrawHill, New York, NY, 2000.

5 S. Ebnesajjad and A. H. Landrock, Adhesives Technology Handbook, Elsevier, 3rd edn, 2015.
6 Q. Ye, F. Zhou and W. Liu, Bioinspired Catecholic Chemistry for Surface Modification, Chem. Soc. Rev., 2011, 40, 42444258.

7 H. Lee, S. M. Dellatore, W. M. Miller and P. B. Messersmith, Mussel-Inspired Surface Chemistry for Multifunctional Coatings, Science, 2007, 318, 426-430.

8 G. P. Maier, M. V. Rapp, J. H. Waite, J. N. Israelachvili and A. Butler, Adaptive synergy between catechol and lysine promotes wet adhesion by surface salt displacement, Science, 2015, 349(6248), 628-632.

9 J. H. Waite and X. Qin, Polyphosphoprotein from the Adhesive Pads of Mytilus edulis, Biochemistry, 2001, 40, 2887-2893.

10 E. W. Danner, Y. Kan, M. U. Hammer, J. N. Israelachvili and J. H. Waite, Adhesion of Mussel Foot Protein Mefp-5 to Mica: An Underwater Superglue, Biochemistry, 2012, 51, 65116518.

11 S. Seo, S. Das, P. J. Zalicki, R. Mirshafian, C. D. Eisenbach, J. N. Israelachvili, J. H. Waite and B. K. Ahn, Microphase Behavior and Enhanced Wet-Cohesion of Synthetic Copolyampholytes Inspired by a Mussel Foot Protein, J. Am. Chem. Soc., 2015, 137, 9214-9217.

12 W. Wei, J. Yu, M. A. Gebbie, Y. Tan, N. R. Martinez Rodriguez, J. N. Israelachvili and J. H. Waite, Bridging Adhesion of Mussel-Inspired Peptides: Role of Charge, Chain Length, and Surface Type, Langmuir, 2015, 31, 11051112.

13 V. Vreeland, J. H. Waite and L. Epstein, Polyphenols and Oxidases in Substratum Adhesion by Marine Algae and Mussels, J. Phycol., 1998, 34, 1-8.

14 Q. Lin, D. Delphine Gourdon, C. Chengjun Sun, N. H. Andersen, T. H. Anderson, J. H. Waite and J. N. Israelachvili, Adhesion Mechanisms of the Mussel Foot Proteins mfp-1 and mfp-3, PNAS, 2007, 104, 3782-3786.

15 M. Yu, J. Hwang and T. J. Deming, Role of L-3,4Dihydroxyphenylalanine in Mussel Adhesive Proteins, $J$. Am. Chem. Soc., 1999, 121, 5825-5826.

16 W. Wei, J. Yu, C. Broomell, J. N. Israelachvili and J. H. Waite, Hydrophobic Enhancement of Dopa-Mediated Adhesion in a Mussel Foot Protein, J. Am. Chem. Soc., 2013, 135, 377-383.

17 T. H. Anderson, J. Yu, A. Estrada, M. U. Hammer, J. H. Waite and J. N. Israelachvili, The Contribution of DOPA to Substrate-Peptide Adhesion and Internal Cohesion of Mussel-Inspired Synthetic Peptide Films, Adv. Funct. Mater., 2010, 20, 4196-4205.

18 J. Monahan and J. J. Wilker, Cross-Linking the Protein Precursor of Marine Mussel Adhesives: Bulk Measurements and Reagents for Curing, Langmuir, 2004, 20, 3724-3729.

19 R. J. Stewart, T. C. Ransom and V. Hlady, Natural Underwater Adhesives, J. Polym. Sci., Part B: Polym. Phys., 2011, 49, 757771.

20 J. H. Waite, N. H. Andersen, S. Jewhurst and C. Sun, Mussel Adhesion: Finding the Tricks Worth Mimicking, J. Adhes., 2005, 81, 297-317.

21 J. J. Wilker, Polymer Composition and Substrate Influences on the Adhesive Bonding of a Biomimetic, Cross-Linking Polymer, J. Am. Chem. Soc., 2012, 134, 9498-9505. 
22 H. J. Meredith and J. J. Wilker, The Interplay of Modulus, Strength, and Ductility in Adhesive Design Using Biomimetic Chemistry, Adv. Funct. Mater., 2015, 25, 50575067.

23 K. Huang, B. P. Lee, D. R. Ingram and P. B. Messersmith, Synthesis and Characterization of Self-Assembling Block Copolymers Containing Bioadhesive End Groups, Biomacromolecules, 2002, 3, 397-406.

24 B. P. Lee, J. L. Dalsin and P. B. Messersmith, Synthesis and Gelation of DOPA-Modified Poly(ethylene glycol) Hydrogels, Biomacromolecules, 2002, 3, 1038-1047.

25 H. Xu, J. Nishida, W. Ma, H. Wu, M. Kobayashi, H. Otsuka and A. Takahara, Competition between Oxidation and Coordination in Cross-Linking of Polystyrene Copolymer Containing Catechol Groups, ACS Macro Lett., 2012, 1, 457-460.

26 M. Yu and T. J. Deming, Synthetic Polypeptide Mimics of Marine Adhesives, Macromolecules, 1998, 31, 4739-4745.

27 H. Yamamoto, T. Ogawa and A. Nishida, Molecular Weight Dependence of Wettability and Molecular Adsorption of Poly-L-lysine at the Air-Water Interface, J. Colloid Interface Sci., 1995, 176, 105-110.

28 D. Kaneko, S. Wang, K. Matsumoto, S. Kinugawa, K. Yasaki, D. H. Chi and T. Kaneko, Mussel-mimetic Strong Adhesive Resin from Bio-base Polycoumarates, Polym. J., 2011, 43, 855-858.

29 J. D. White and J. J. Wilker, Underwater Bonding with Charged Polymer Mimics of Marine Mussel Adhesive Proteins, Macromolecules, 2011, 44, 5085-5088.

30 K. Yamada, T. Chen, G. Kumar, O. Vesnovsky, L. D. T. Topoleski and G. F. Payne, Chitosan Based WaterResistant Adhesive. Analogy to Mussel Glue, Biomacromolecules, 2000, 1, 252-258.

31 G. Westwood, T. N. Horton and J. J. Wilker, Simplified Polymer Mimics of Cross-Linking Adhesive Proteins, Macromolecules, 2007, 40, 3960-3964.

32 H. J. Meredith, C. L. Jenkins and J. J. Wilker, Enhancing the Adhesion of a Biomimetic Polymer Yields Performance Rivaling Commercial Glues, Adv. Funct. Mater., 2014, 24, 3259-3267.

33 H. K. Reimschuessel, On the Glass Transition Temperature of Comblike Polymers: Effects of Side Chain Length and Backbone Chain Structure, J. Polym. Sci., Part A: Polym. Chem., 1979, 17(8), 2447-2457.

34 V. M. Choudhary and A. Mishra, Studies on the Copolymerization of Methyl Methacrylate and N-Aryl maleimides, J. Appl. Polym. Sci., 1996, 62, 707-712.

35 M. Hisano, K. Takeda, T. Takashima, Z. Z. Jin, A. Shiibashi and A. Matsumoto, Sequence-Controlled Radical
Copolymerization of N-Substituted Maleimides with Olefins and Polyisobutene Macromonomers To Fabricate Thermally Stable and Transparent Maleimide Copolymers with Tunable Glass Transition Temperatures and Viscoelastic Properties, Macromolecules, 2013, 46, 77337744 .

36 S. S. Thamizharasi and B. S. R. Reddy, Copolymerization of N-Substituted Maleimide with Alkyl Acrylate and Its Industrial Applications, J. Appl. Polym. Sci., 2001, 80, 18701879.

37 J. Brandrup, E. H. Immergut and E. A. Grulke, Polymer Handbook, John Wiley and Sons, Inc., New York, 4th edn, 1999.

38 L. E. Coleman and J. A. Conrady, Nitrogen-Containing Monomers. I. Copolymerization Reactions of $N$-Alkyl Maleamic Acids and $N$-Alkyl Maleimides, J. Polym. Sci., 1959, 38, 241-245.

39 Y. Nakayama and G. Smets, Radical and Anionic Homopolymerization of Maleimide and N-nButylmaleimide, J. Polym. Sci., 1967, 5, 1619-1633.

40 L. J. Fetters, D. J. Lohse and R. H. Colby, Chain Dimensions and Entanglement Spacings, in Physical Properties of Polymers Handbook, ed. J. E. Mark, Springer New York, New York, NY, 2007; pp. 447-454.

41 L. J. Fetters, D. J. Lohse, D. Richter, T. A. Witten and A. Zirkelt, Connection between Polymer Molecular Weight, Density, Chain Dimensions, and Melt Viscoelastic Properties, Macromolecules, 1994, 27, 4639-4647.

42 M. Krogsgaard, M. A. Behrens, J. S. Pedersen and H. Birkedal, Self-Healing Mussel-Inspired Multi-pHResponsive Hydrogels, Biomacromolecules, 2013, 14, 297301.

43 J. Yu, W. Wei, M. S. Menyo, A. Masic, J. H. Waite and J. N. Israelachvili, Adhesion of Mussel Foot Protein-3 to $\mathrm{TiO}_{2}$ Surfaces: the Effect of pH, Biomacromolecules, 2013, 14, 1072-1077.

44 The anticipated changes in the thermal transitions were garnered from pure polymethylacrylate and polyhexylacryalte which have a $67{ }^{\circ} \mathrm{C}$ difference in $T_{\mathrm{g}}$ according to ref. 33 .

45 K. Kunal, C. G. Robertson, S. Pawlus, S. F. Hahn and A. P. Sokolov, Role of Chemical Structure in Fragility of Polymers: A Qualitative Picture, Macromolecules, 2008, 41, 7232-7238.

46 J. M. Torres, C. Q. Wang, E. B. Coughlin, J. P. Bishop, R. A. Register, R. A. Riggleman, C. M. Stafford and B. D. Vogt, Influence of Chain Stiffness on Thermal and Mechanical Properties of Polymer Thin Films, Macromolecules, 2011, 44, 9040-9045. 